\title{
Rotating elephant trunks ${ }^{\star}$
}

\author{
G. F. Gahm ${ }^{1}$, P. Carlqvist ${ }^{2}$, L. E. B. Johansson ${ }^{3}$, and S. Nikolić ${ }^{4,5}$ \\ 1 Stockholm Observatory, AlbaNova University Centre, 10691 Stockholm, Sweden \\ e-mail: gahm@astro.su.se \\ 2 Alfvén Laboratory, Royal Institute of Technology, 10044 Stockholm, Sweden \\ 3 Onsala Space Observatory, 43992 Onsala, Sweden \\ ${ }^{4}$ Departamento de Astronomía, Universidad de Chile, Casilla 36D, Santiago, Chile \\ 5 Astronomical Observatory, Volgina 7, 11060 Belgrade, Serbia and Montenegro
}

Received 8 November 2005 / Accepted 26 January 2006

\section{ABSTRACT}

\begin{abstract}
Aims. We investigate the structure and velocity of cold molecular pillars, "elephant trunks", in expanding H II regions.
Methods. The trunks are seen in silhouette against the bright background in our $\mathrm{H} \alpha$ images. All trunks are filamentary, and show signs of being twisted. Four such trunks in NGC 7822, IC 1805, the Rosette Nebula, and DWB 44 were selected, and then mapped mainly in ${ }^{12} \mathrm{CO}$ and ${ }^{13} \mathrm{CO}$. We determine the mass and density of the trunks. Most of the mass is concentrated in a head facing the central cluster, and in sub-filaments forming the body of the trunk that is connected to $V$-shaped filaments to the outer expanding shell.

Results. We discovered that all four trunks rotate as rigid bodies (to a first approximation) about their major axes, and that at least two trunks are stretching along their major axes, meaning that the massive heads are lagging behind in the general expansion of the H II regions. The rotational periods are of the order of a few million years - similar to the age of the clusters. Rotation, then, is responsible for the twisted appearance of many elephant trunks, since they are rooted in the outer shells. The trunks carry surprisingly large amounts of angular momentum, $3 \times 10^{48}-2 \times 10^{50} \mathrm{~kg} \mathrm{~m}^{2} \mathrm{~s}^{-1}$, with corresponding rotational energies of up to $\sim 10^{37} \mathrm{~J}$. However, we estimate the total magnetic energies to be even larger. The trunks continuously reshape, and the formation of twined, and in many cases helical, sub-filaments can be understood as a consequence of electromagnetic and inertia forces inside the trunks. A theory based on the concept of magnetically twisted trunks is developed further, where the initial angular momentum is a consequence of the twisting of parent filaments containing mass condensations. Our results also suggest a new process of removing angular momentum from parent molecular clouds.
\end{abstract}

Key words. ISM: H II regions - ISM: clouds - ISM: kinematics and dynamics - ISM: magnetic fields

\section{Introduction}

The HII regions contain dusty gas, which in general is organised in networks of filaments. In many regions the dark dust lanes are seen as silhouettes on the background of bright nebulosity on optical images. These elongated structures often point like fingers towards hot stars located in the centre of the nebulae, and such lanes are called elephant trunks. Examples of elephant trunks have been presented by e.g. Minkowski (1949), Gyul'budagyan \& Akopyan (1990), Pottasch (1956), Gyul'budagyan et al. (1994), and Ogura \& Sugitani (1998). Other examples and discussions of their nature can be found in e.g. Osterbrock (1957), Menon (1962), Herbig (1974), Schneps et al. (1980), Hester et al. (1996), Carlqvist et al. (1998, hereafter called CKG98), White et al. (1999) and Pound et al. (2003).

The first theoretical models of the origin and structure of elephant trunks were based on the Rayleigh-Taylor instability (Spitzer 1954; Frieman 1954), later modified to include the action from stellar winds (e.g. Pound 1998). Stellar winds also play an important role in a different theory of trunks developed by Pikel'ner (1973) and Pikel'ner \& Sorochenko (1974), where shells are driven outwards through surrounding cold gas containing clumps. These will accelerate more slowly than the surrounding, and long, extended structures pointing inwards

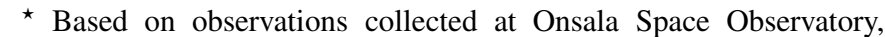
Sweden, and Nordic Optical Telescope, La Palma, Spain. will develop. In other variations on this theme ionization-shock fronts have been considered (Giuliani 1979), as well as combinations of photo-ionization and winds (Garcia-Segura \& Franco 1996).

We have carried out an $\mathrm{H} \alpha$ survey of $10 \mathrm{H}$ II regions with trunks using the Nordic Optical Telescope (NOT). As is evident from these images, as well as from a number of images obtained with the Hubble Space Telescope of similar objects, the trunks often have a very intriguing internal structure containing long, thin filaments and knots (see Gahm 2003). An interesting finding is that many filaments show evidence of a twisted or helical structure. The twisted geometry of the trunks is not accounted for in the previous theoretical models, but an outline of a new theory of such trunks has recently been advanced by Carlqvist et al. (2002, hereafter called CGK02). The theory is based on the presence of magnetized filaments (magnetic ropes) in molecular clouds where H II regions are formed through the action of young, hot stars. If a filament is swept up by the expanding $\mathrm{H}$ II region, it may under certain conditions be twisted into a double helix that points at the hot stars. The theory was further developed by Carlqvist et al. (2003, hereafter called CGK03), where more examples of NOT images of twisted trunks are also presented.

The twisted trunks considered for this first-order model are in equilibrium, but at an earlier phase twinning has occurred, so rotation of cold filamentary cloudlets is expected to take place. 
Table 1. Basic data for the regions observed.

\begin{tabular}{lccccl}
\hline \hline & NGC 7822 & IC 1805 & Rosette & DWB 44 & Ref. \\
\hline Distance $d$ [pc] & 850 & 2350 & 1400 & 740 & $1-7$ \\
Bubble radius [pc] & 12 & 26 & 16 & 7.5 & $8-15$ \\
Age of cluster [yrs/10 $]$ & 2 & 2 & 4 & - & $1,4-6,16$ \\
Central velocity $\left[1\right.$. s.r., $\mathrm{km} \mathrm{s}^{-1}$ ] & -15.7 & -46 & +16.7 & - & $13,17,18$ \\
\hline
\end{tabular}

References: 1. MacConnell (1968); 2. Guetter \& Vrba (1989); 3. Basu et al. (1999); 4. This work; 5. Massey et al. (1995); 6. Ogura \& Ishida (1981); 7. Gyul'budagyan et al. (1994); 8. Pedlar (1980); 9. Harten et al. (1981); 10. Normandeau et al. (1997); 11. Tereby et al. (2003); 12. Graham et al. (1982); 13. Celnik (1985); 14. Dickel \& Wendker (1977); 15. Wendker et al. (1991); 16. Cohen \& Kuhi (1976);17. Liu et al. (1988); 18. Georgelin \& Georgelin (1970).

The question is whether some trunks are in fact rotating. We undertook a study of the radio molecular line emission from trunks at higher spatial resolution than used before, in order to map the velocity fields in the trunks, and to look for signs of rotation. From our NOT survey we selected a number of interesting trunks, and were able to observe four of these in great detail.

\section{Observations and objects}

The mm-wave observations were done during three sessions between January and September, 2003, using the $20 \mathrm{~m}$ telescope of the Onsala Space Observatory. The basic data set consists of maps in the ${ }^{12} \mathrm{CO}(1-0)$ and ${ }^{13} \mathrm{CO}(1-0)$ transitions done in the frequency-switching mode. The frontend was a SIS mixer with a typical receiver temperature of $100 \mathrm{~K}$ (SSB). We used a 1600 channel correlator with a band width of $40 \mathrm{MHz}$ implying a velocity resolution of about $0.07 \mathrm{~km} \mathrm{~s}^{-1}$. The pointing was checked regularly, and we estimate the total blind pointing error to have been less than $4^{\prime \prime} \mathrm{rms}$, while the relative error of grid points within each map should be a factor two less. At $110 \mathrm{GHz}$ the $H P B W$ is $33^{\prime \prime}$, and the main beam efficiency is 0.6 . The online intensity scale $\left(T_{\mathrm{A}}^{*}\right)$ is defined by the chopper-wheel method. To derive physical parameters of the regions observed we used main-beam brightness temperature.

For the collection of $\mathrm{H} \alpha$ images we used the ALFOSC camera with a narrow-band $\mathrm{H} \alpha$ filter attached to the $2.6 \mathrm{~m}$ NOT. The field of view is 6.5 , and the effective scale of the CCD detector is 0.188 per pixel. The exposure times were typically $30 \mathrm{~min}$. From stars in the fields we measured angular resolutions in the range $0 .{ }^{\prime} 7-1$ '. 0 . Bias, dark current, and flat field corrections were applied, as well as corrections for the sky background as extracted from sky field exposures.

Our collection of images contain a large number of elephant trunks, and we were able to complete radio observations of the four rather prominent trunks described below. They are located in the four $\mathrm{H}$ II regions listed in Table 1 with some basic data extracted from the literature. The radius of a given $\mathrm{H}$ II region was estimated primarily from radio continuum maps of the region, but also from $\mathrm{H} \alpha$ images of the area.

$N G C 7822(S 171, W 1)$ is connected to the Cep OB4 association, centred on the young cluster Berkeley 59 in a large molecular cloud containing bright rimmed features (Sugitano et al. 1991; Ogura et al. 2002). The region has been mapped in several molecular lines, showing that several velocity components are present at certain locations (see e.g. Rydbeck et al. 1976; and Leisavitz et al. 1989).

We call the elephant trunk selected for this study the Dancing Queen, labelled in the following the $D Q$ Trunk. It points in the direction of $\mathrm{Be} 59, \sim 18^{\prime}$ to the north, and is shown in Fig. 1, where the $(0,0)$ position for the radio mapping is at $\alpha=00^{\mathrm{h}} 02^{\mathrm{m}} 12.6^{\mathrm{s}}, \delta=+67^{\circ} 06^{\prime} 47^{\prime \prime}(\mathrm{J} 2000)$, and the map grid,

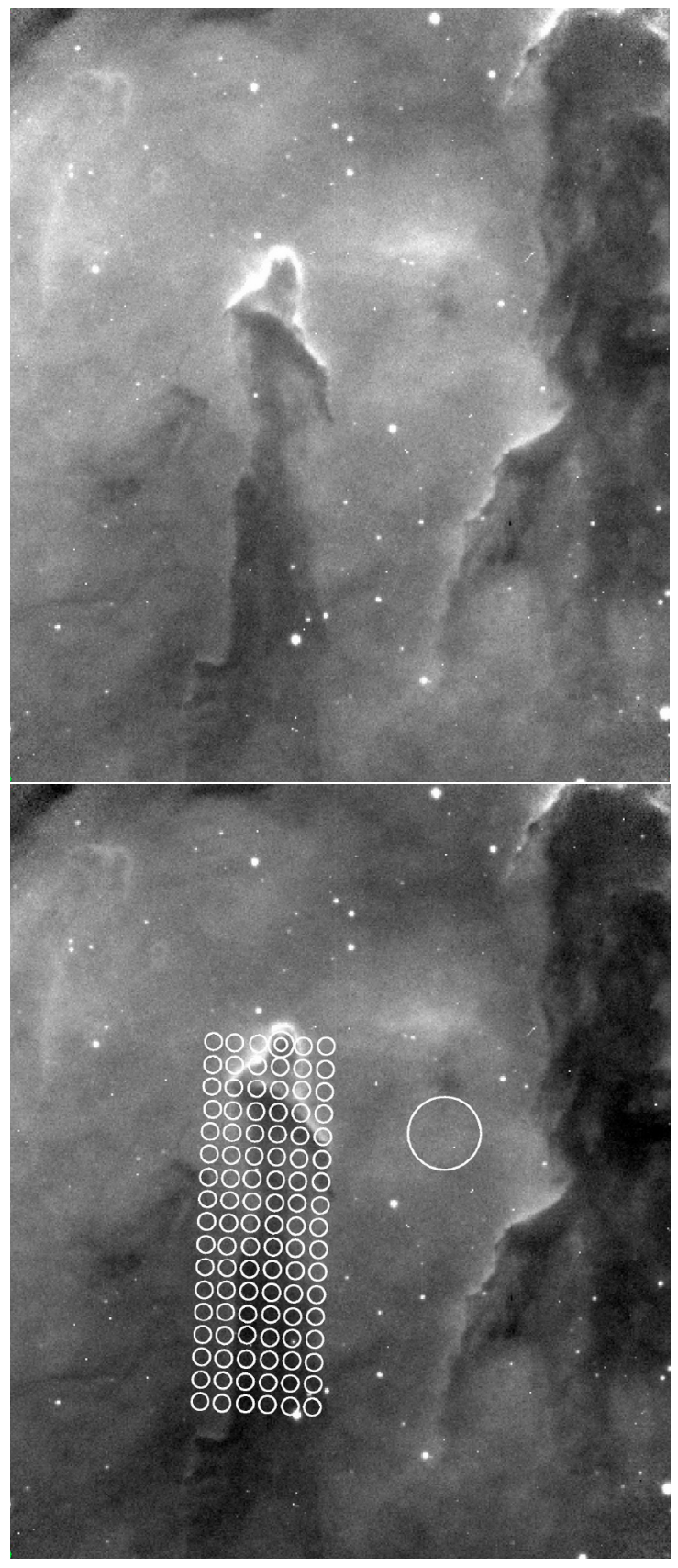

Fig. 1. The elephant trunk "the Dancing Queen" in the NGC 7822 complex has a bright rimmed head, which is broken into an extended wisp. The $\mathrm{H} \alpha$ images span 4.8 $\times 5.7$, and in the lower panel, the positions observed for molecular emission are shown with the $(0,0)$ position marked as a double circle. The grid size is $10^{\prime \prime} \times 10^{\prime \prime}$. The large circle indicates the beam size. Another more massive trunk, also with a broken top, is seen to the right (north: up; east: left). 


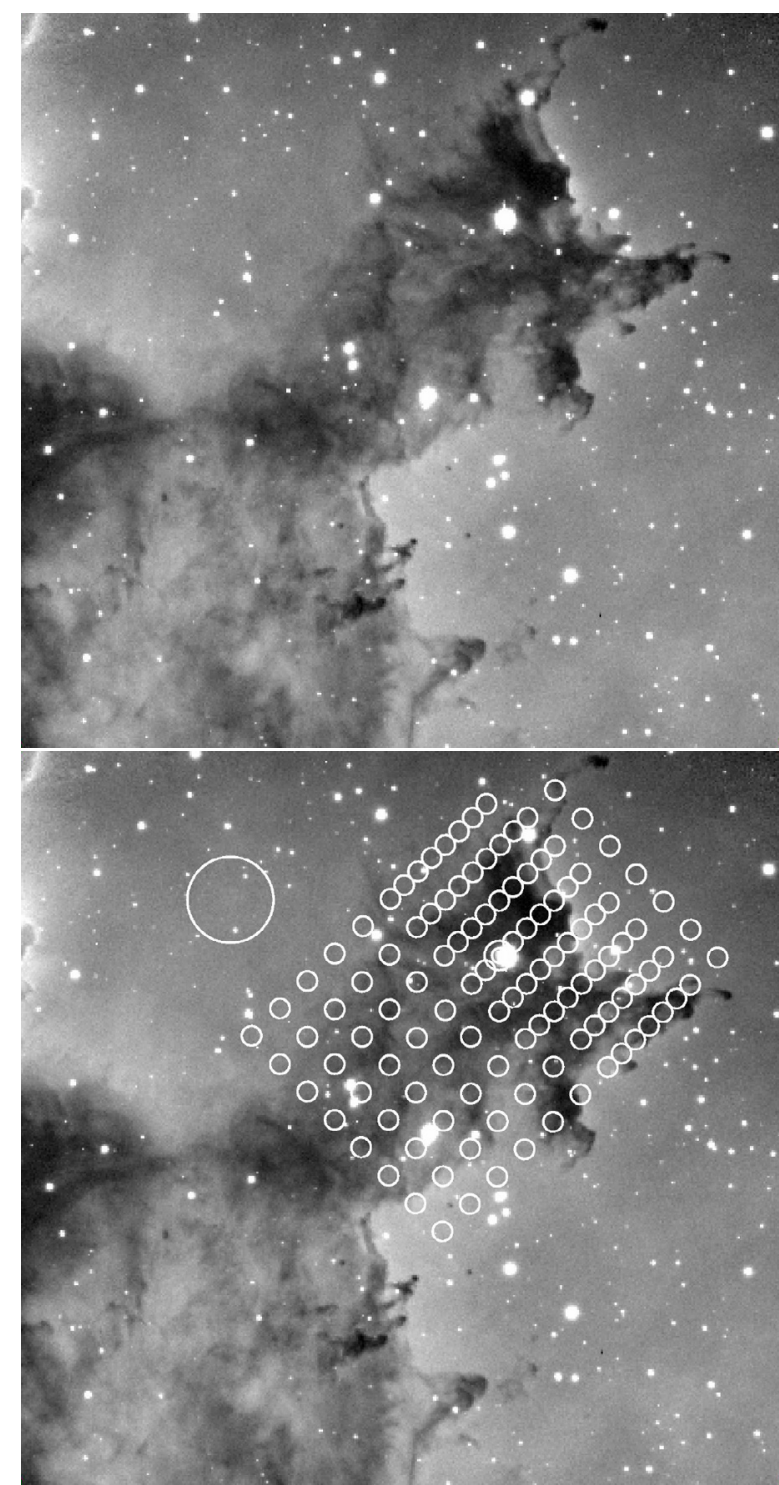

Fig. 2. The "Stag-Beetle" is a very large elephant trunk in the IC 1805 complex (W4). The top is jaw-like, and composed of twisted threads. The $\mathrm{H} \alpha$ images span $4^{\prime} 8 \times 4^{\prime} .7$. The grid size is $15^{\prime \prime} \times 15^{\prime \prime}$, with steps of 7". 5 across the top. Symbols as in Fig. 1.

with a spacing of $10^{\prime \prime}$, was tilted with a position angle of $-2^{\circ}$ in accordance with the corresponding slight tilt of the trunk with respect to the north-south direction. All grid positions were mapped in the $(1-0)$ transitions of ${ }^{12} \mathrm{CO}$ and ${ }^{13} \mathrm{CO}$, and the region around the "head" was also observed for $\mathrm{CS}$ and $\mathrm{HCO}^{+}$.

IC 1805 (S 171, W4) is connected to the association Cas OB6 including the young cluster $\mathrm{OCl} 352$. The complex is part of a chain of prominent $\mathrm{H}$ II regions connected to massive molecular clouds in the Perseus arm.

The trunk mapped is shown in Fig. 2. This is an unusually large trunk with a projected size of $0.9 \times 2.2$ pc that was discussed in some detail by CGK03, who named it the Stag-Beetle (referred to below as the $S B$ Trunk). The major axis of the trunk points in a direction more than $20^{\circ}$ to the north of the core of $\mathrm{OCl}$ 352. The trunk is located in the interface between W4 and a superbubble ("chimney") expanding in the direction north from OCl 352 as described by Taylor et al. (1999), Basu et al. (1999), and Tereby et al. (2003). The orientations of trunks in the area can be affected by this additional dynamical structures, and/or from the recent passage through a galactic shock, as discussed by Dickel et al. (1980). OCl 352 has a pronounced proper motion as discussed by Dambis et al. (2001) and Mirabel et al. (2004), and we note that the Stag-Beetle is pointing in a direction where one expects the cluster to have been located $\sim 7 \times 10^{5}$ years ago. The question is whether the trunk was sculptured and oriented prior to this early phase but in a surrounding shell not moving with the cluster.

Previous mappings of CO emission by e.g. Lada et al. (1978) and Carpenter (2000) show no detections in the area in question, and in ${ }^{13} \mathrm{CO}$ we only obtained reasonable signals from the jaw. We have therefore focussed on ${ }^{12} \mathrm{CO}$, which was observed according to the grid shown in Fig. 2 and with spacings of $7 " .5$ and $15^{\prime \prime}$ along the axis of the trunk and $15^{\prime \prime}$ across this axis. The $(0,0)$ position is at $\alpha=02^{\mathrm{h}} 36^{\mathrm{m}} 30.0^{\mathrm{s}}, \delta=+61^{\circ} 23^{\prime} 46^{\prime \prime}(\mathrm{J} 2000)$, and the map grid was tilted by $-45^{\circ}$.

The Rosette Nebula (NGC 2237 - 2246), where NGC 2244 is the central cluster, is a magnificent, expanding H II region surrounded by a network of cold, molecular filaments containing a number of elephant trunks and drop-shaped globules, as noted already by Minkowski (1949) and Bok et al. (1949).

For the present study we selected one very prominent elephant trunk, which we call the Wrench (here labelled the $W$ Trunk) and which we have studied before in detail (CKG98, CGK02, CGK03). We collected several NOT images at different positions over the area in question. The trunk is composed of bundles of thin, twisted filaments, which appear to be organized in helical shapes (see Fig. 3). These filaments connect the more massive "head" to the nebular surrounding. The head has a jawlike appearance. A rather detailed mapping of ${ }^{12} \mathrm{CO}$ and ${ }^{13} \mathrm{CO}$ of the trunk and its surrounding was made by Schneps et al. (1980) with a beam of 1 '. 1 , and typical spacings of $1^{\prime}$. This study demonstrated that the trunk as a whole is moving outwards with respect to the central cluster to which the trunk is pointing, They also found that the trunk is stretching and expanding along its major axis.

We mapped the $W$ Trunk in ${ }^{12} \mathrm{CO}$ over a grid tilted by $-12^{\circ}$, which is shown in Fig. 3. The $(0,0)$ position is at $\alpha=$ $06^{\mathrm{h}} 31^{\mathrm{m}} 36.0^{\mathrm{s}}, \delta=+05^{\circ} 08^{\prime} 00^{\prime \prime}(\mathrm{J} 2000)$. The jaw was covered in steps of $10^{\prime \prime}$, while the extended feature to the north of the jaw was scannedalso observed over the same grid in ${ }^{13} \mathrm{CO}$, while only a few scans were made across the filaments to the north since it turned out that here the signal is very weak.

$D W B 44$ is one of many $\mathrm{H}$ II regions listed by Dickel et al. (1969) in the so-called Cygnus X complex, a region of the local spiral arm that we view tangentially. The distances to these nebulae are poorly known, but Gyul'budagyan et al. (1994) suggests that several are related to the Cyg OB 9 association, for which they assigned a distance of 740 pc. For DWB 44 no exciting star has been recognised so far.

DWB 44 was selected because it contains a prominent elephant trunk where the head forms an extremely complex, open, jaw-like structure. This jaw is connected to the nebular surrounding with two filamentary legs in a $V$-shaped formation (see Fig. 4). We name this trunk the Batman (labelled the $B$ Trunk) from the appearance of the head. The $(0,0)$ position of the ${ }^{13} \mathrm{CO}$ map is at $\alpha=20^{\mathrm{h}} 18^{\mathrm{m}} 42.2^{\mathrm{s}}, \delta=+39^{\circ} 37^{\prime} 12^{\prime \prime}$ (J2000). The map grid, with a spacing of $10^{\prime \prime}$, was tilted with a position angle of $-21^{\circ}$ (see Fig. 4). We also mapped the central part of the jaw in ${ }^{12} \mathrm{CO}$. 


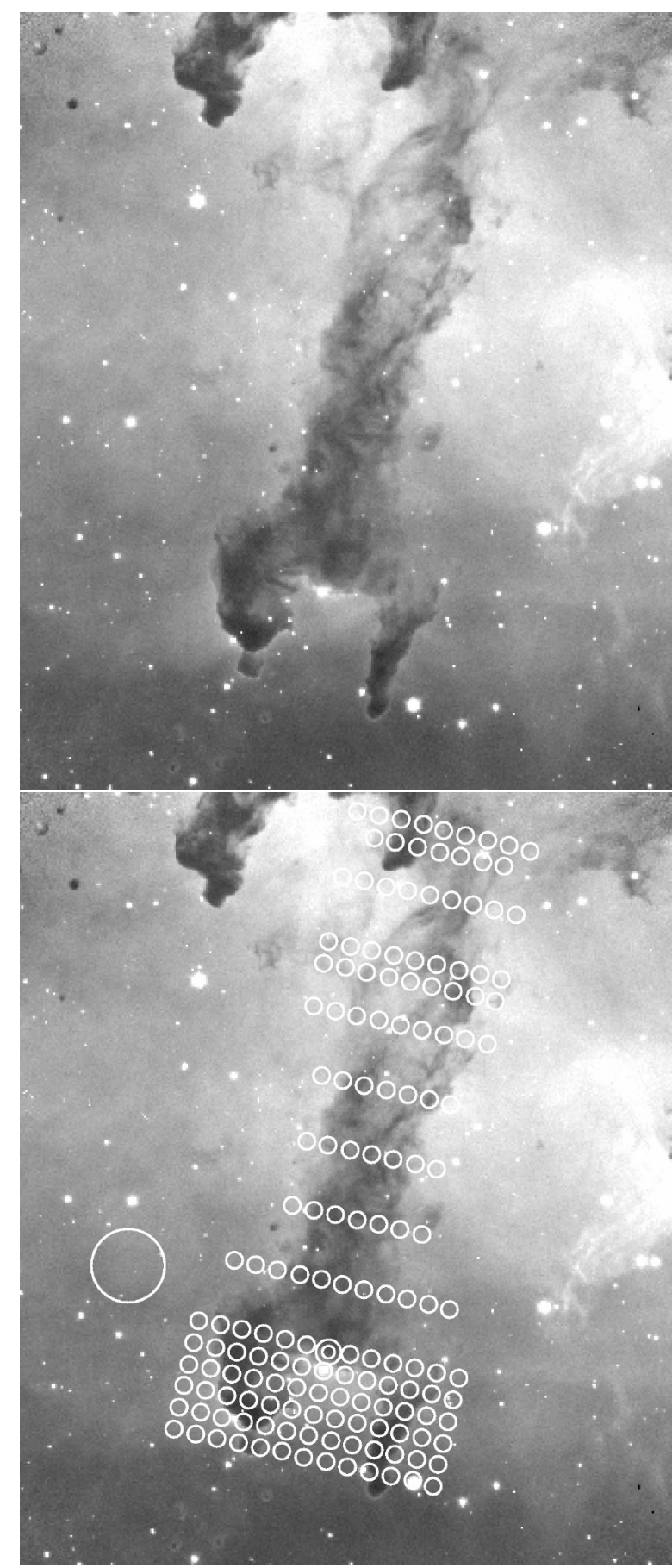

Fig. 3. The "Wrench" Trunk in the Rosette Nebula is composed of threads in a helix-like configurations and a jaw. The trunk has several systems of connected globules. The $\mathrm{H} \alpha$ images span $4.9^{\prime} \times 5.8^{\prime}$. The grid size is $10^{\prime \prime} \times 10^{\prime \prime}$ over the jaw. Symbols as in Fig. 1 .

\section{Results}

The line emission connected to the trunks could easily be identified since no emission - or at most weak - is present at the off-source positions. The individual observations were first ordered in maps like the one in Fig. 5 of ${ }^{12} \mathrm{CO}$ of the $W$ Trunk, showing intensity versus velocity. The line profiles are as a rule close to Gaussian in shape in all regions with little evidence of any pronounced additional components. As an example we show the $\mathrm{HCO}^{+}$line profiles of the "head" of the $D Q$ Trunk in Fig. 6 . In addition, maps of integrated intensity, central velocity, and

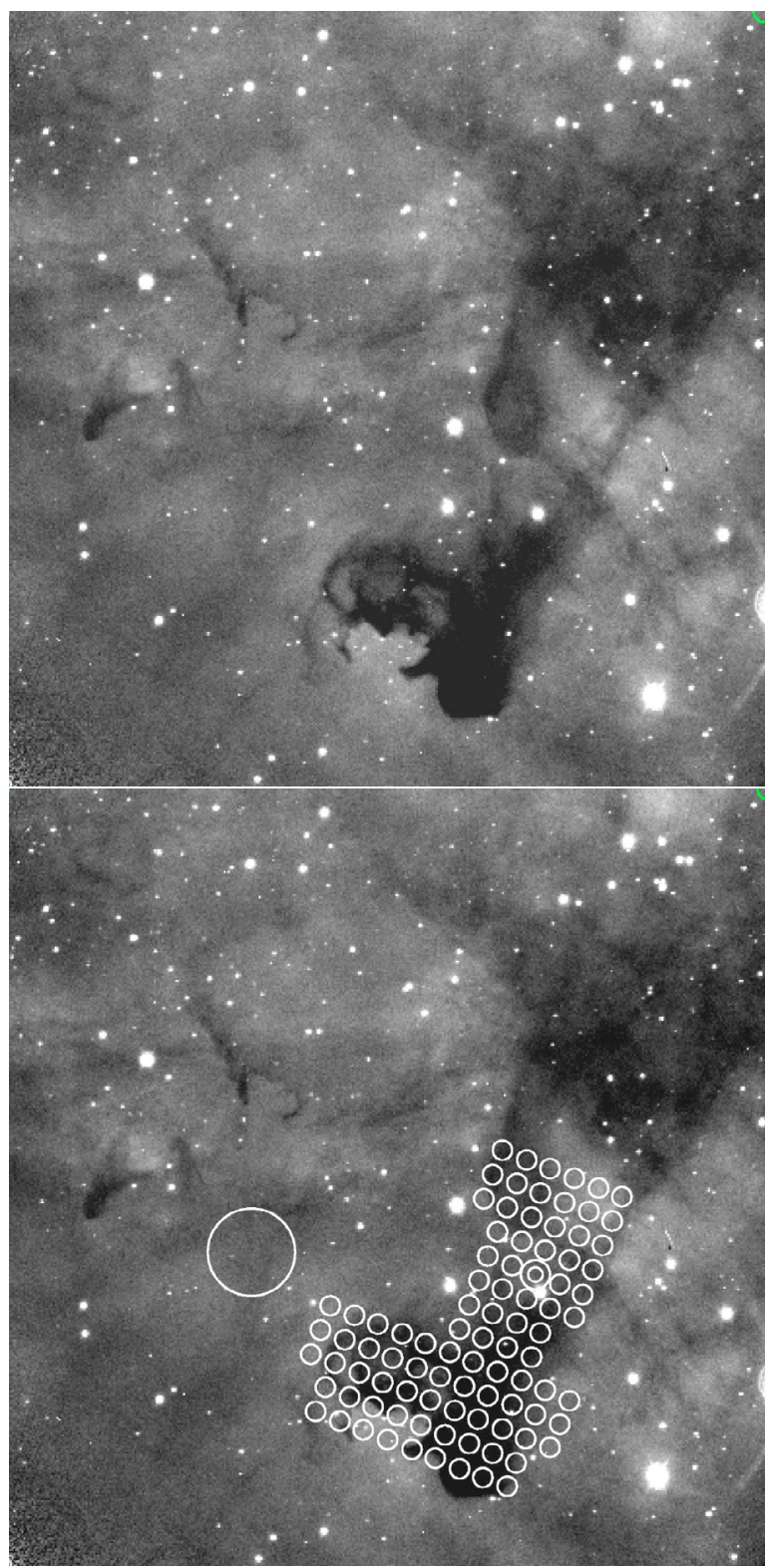

Fig. 4. We call this trunk "the Batman" from the appearance of the complex system of threads in the jaw-like head. It may be related to the H II region DWB 44 and the Cyg OB9 association. The images span $4^{\prime} .9 \times 5^{\prime} 1$. The grid size is $10^{\prime \prime} \times 10^{\prime \prime}$. Symbols as in Fig. 1 .

line width (FWHM) were derived from Gaussian fitting of the line profiles. Figure 7 shows the integrated intensity map of the $D Q$ Trunk indicating the presence of two main mass condensations, one related to the "head" and one to the "body" of the trunk. In contrast, most of the mass in the $B$ Trunk is concentrated in the head as seen in the ${ }^{13} \mathrm{CO}$ integrated intensity map in Fig. 8. The ${ }^{13} \mathrm{CO}$ central velocity map of the $W$ Trunk is shown in Fig. 9, which reveals the presence of a smooth velocity gradient component across this part of the trunk. The integrated intensity maps give a clue to the orientation of the trunks in the sky plane. We find that the pre-selected position angles for the radio grids are in good agreement with what can be inferred from the CO maps.

An important finding is that there are regions in all the trunks studied where the residual intensity is much less than $50 \%$ of the bright background on the NOT images. This would not occur if a trunk were located on the remote side of a relatively 


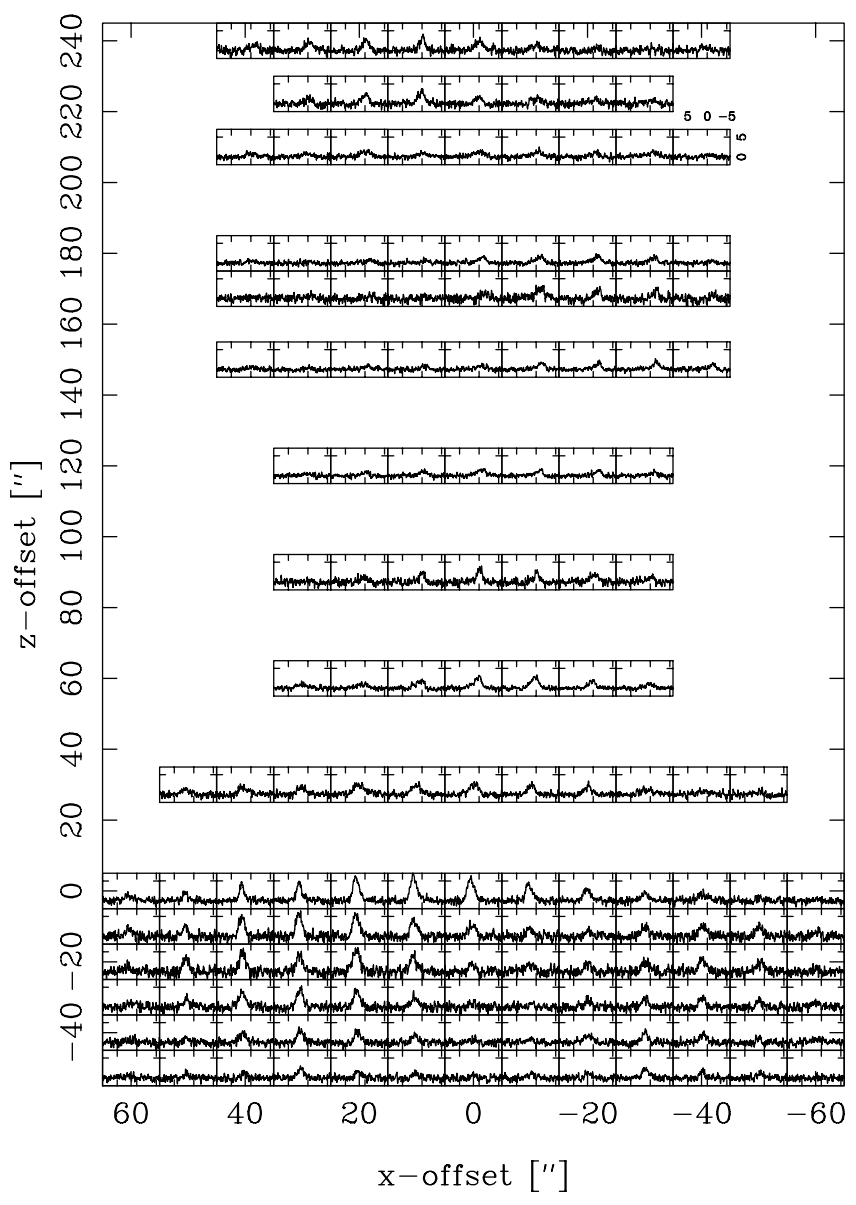

Fig. 5. The panels show ${ }^{12} \mathrm{CO}$ emission intensity $\left(T_{\mathrm{A}}^{*}\right.$-scale) versus local standard velocity $\left(\mathrm{km} \mathrm{s}^{-1}\right)$ over the Wrench Trunk in the Rosette Nebula (see scales in the panel at offsets $-40,210$ ). The $z$-axis shows offsets along the central axis of the tilted trunk (see Fig. 3 for a view of orientations).

homogeneous and regular $\mathrm{H}$ II region producing substantial foreground emission. Furthermore, the $D Q, S B$ and $W$ Trunks all show bright rim features that appear to be dominated by evaporation/excitation of gas on the backside of the trunks. These circumstances lead us to conclude that all our trunks are located on the near side of the ionized bubbles.

\subsection{Velocities}

The upper panel of Fig. 10 depicts the central velocities for different positions in the $D Q$ Trunk. The individual formal errors in central velocity are usually $\leq 0.1 \mathrm{~km} \mathrm{~s}^{-1}$, unless the signal is weak. This trunk shows a distinct velocity gradient component perpendicular to the trunk axis. In addition, there is a pronounced velocity gradient component along the trunk axis. However, the span of velocities for a given off-axis position varies smoothly along the trunk, being smallest at the head. This trunk has the smallest angular diameter in our sample, and the "head" and the upper part of the "body" are each covered by one beam. The lower part of the body is wider, and more diffuse, especially to the west. Hence, beam-filling effects are important in shaping the velocity pattern observed.

To simulate the velocity patterns observed in the trunks, we used a simple model consisting of rigidly rotating, truncated cones of constant gas density. Each cone is defined by its height and radii (lower and upper); the $\mathrm{CO}$ gas parameters

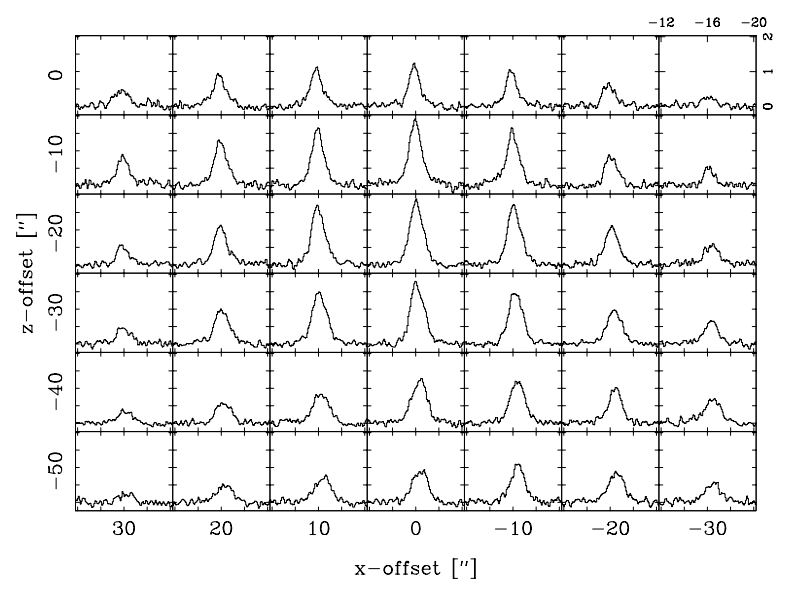

Fig. 6. Map of $\mathrm{HCO}^{+}$profiles over the "head" of the Dancing Queen Trunk in NGC 7822 (axes defined as in Fig. 5). The profiles are nearly Gaussian in shape and lack distinct additional components, for instance at the positions where the foreground "wisp" crosses the head (see Fig. 1).

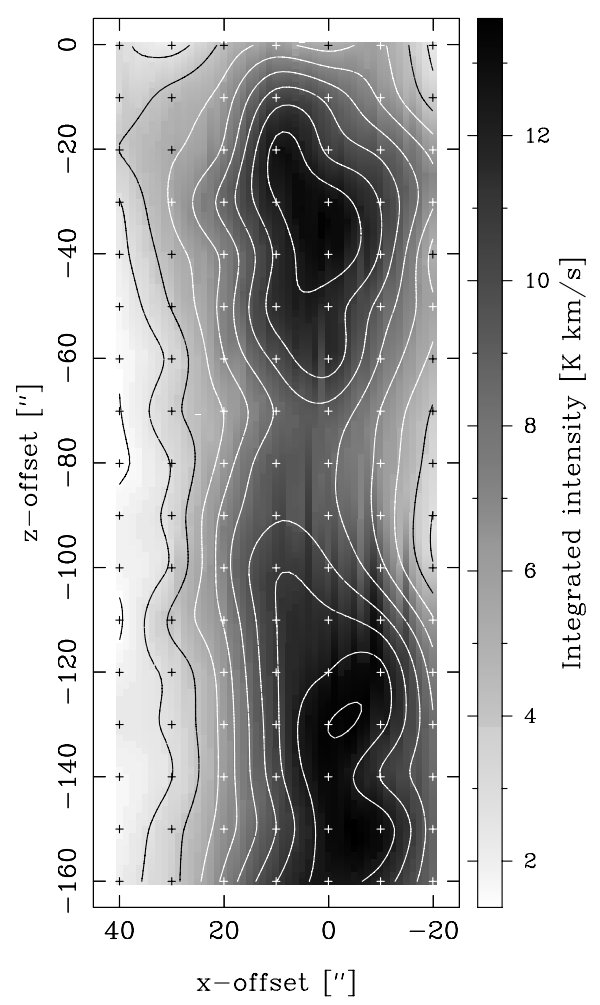

Fig. 7. Integrated intensity $\left(\mathrm{K} \mathrm{km} \mathrm{s}^{-1}\right)$ map of ${ }^{12} \mathrm{CO}$ of the Dancing Queen Trunk (axes defined as in Fig. 5).

are assumed constant, and described by the excitation temperature $\left(T_{\mathrm{ex}}\right)$ and maximum optical depth $\tau_{0}$. Spectra were calculated by a numerical integration algorithm using the simple formula, $T_{\mathrm{A}}=T_{\mathrm{ex}}\left(1-\mathrm{e}^{\tau}\right)$, weighted by the appropriate beam response (assumed to be a Gaussian function).

The $D Q$ Trunk was tuned to fit the observed velocity grid. The model velocities are depicted in the lower panel of Fig. 10 and agree well with the observations. As a result, the observed velocity shifts across the trunk ( $x$-axis), and their change with position along the $z$-axis can be fully understood as a consequence of different beam-filling fractions for the emission from different positions in this narrow trunk assumed to rotate rigidly about the $z$-axis. The true velocity gradient component across 


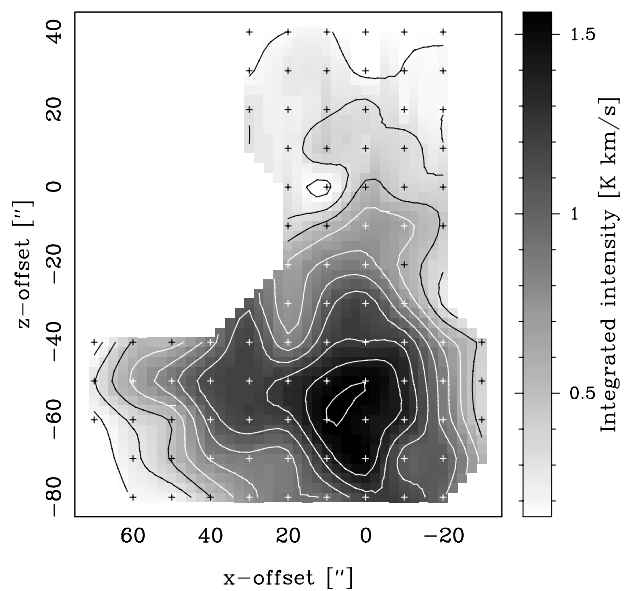

Fig. 8. Integrated intensity map of ${ }^{13} \mathrm{CO}$ of the Batman Trunk in DWB 44 (axes defined as in Fig. 5).

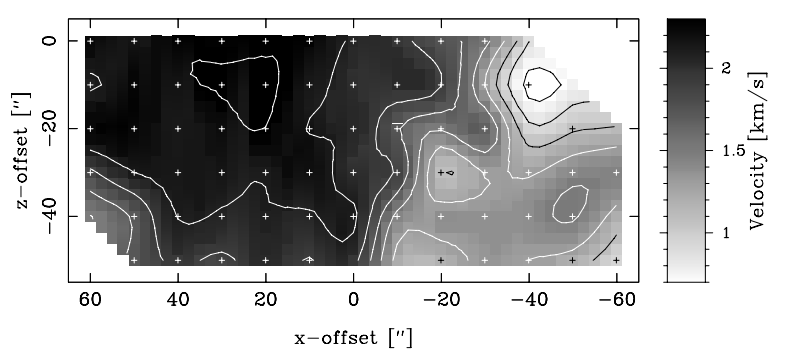

Fig. 9. The ${ }^{13} \mathrm{CO}$ central velocity map of the jaw in the Wrench Trunk indicating the presence of a velocity gradient component across the trunk (axes defined as in Fig. 5).
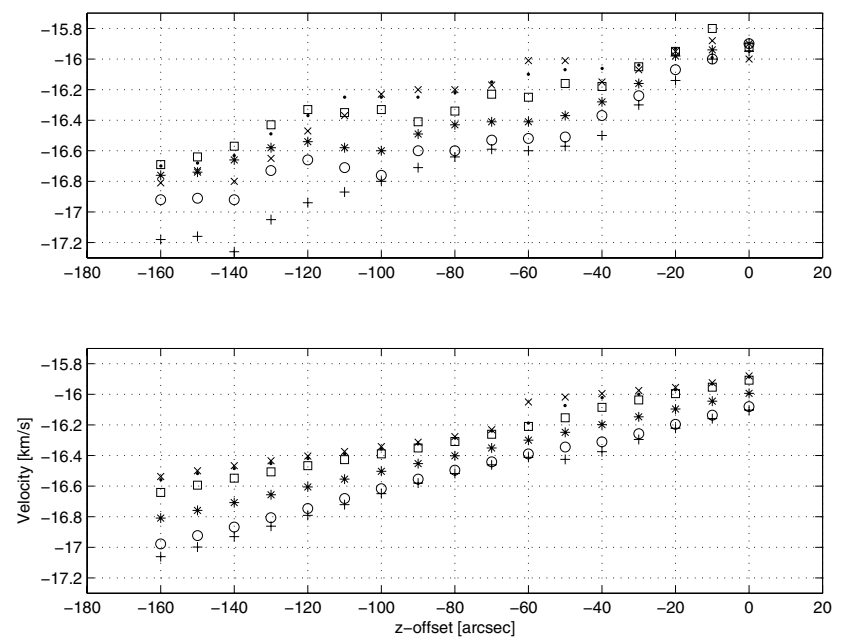

Fig. 10. Top: observed central 1.s.r velocities for the $D Q$ Trunk from individual ${ }^{12} \mathrm{CO}$ line profiles at different offsets in $z$. The different symbols mark the different offsets in $x$ from $-20^{\prime \prime}$ to $+40^{\prime \prime}$ (and follow this order upwards at for instance $\left.z=-60^{\prime \prime}\right)$. The "head" is located at the right end. Bottom: derived velocities over the same grid from a model of a rigidly rotating trunk with a velocity gradient component along the $z$-axis.

the trunk is therefore considerably larger than the observed one, especially over the upper part of the trunk. Superimposed on this velocity pattern is a smooth velocity gradient component along the $z$-axis.

Figure 11 shows the observed velocity patterns for the other three trunks. For ${ }^{13} \mathrm{CO}$, and for other molecules mapped over limited regions, the velocity patterns are congruent with those
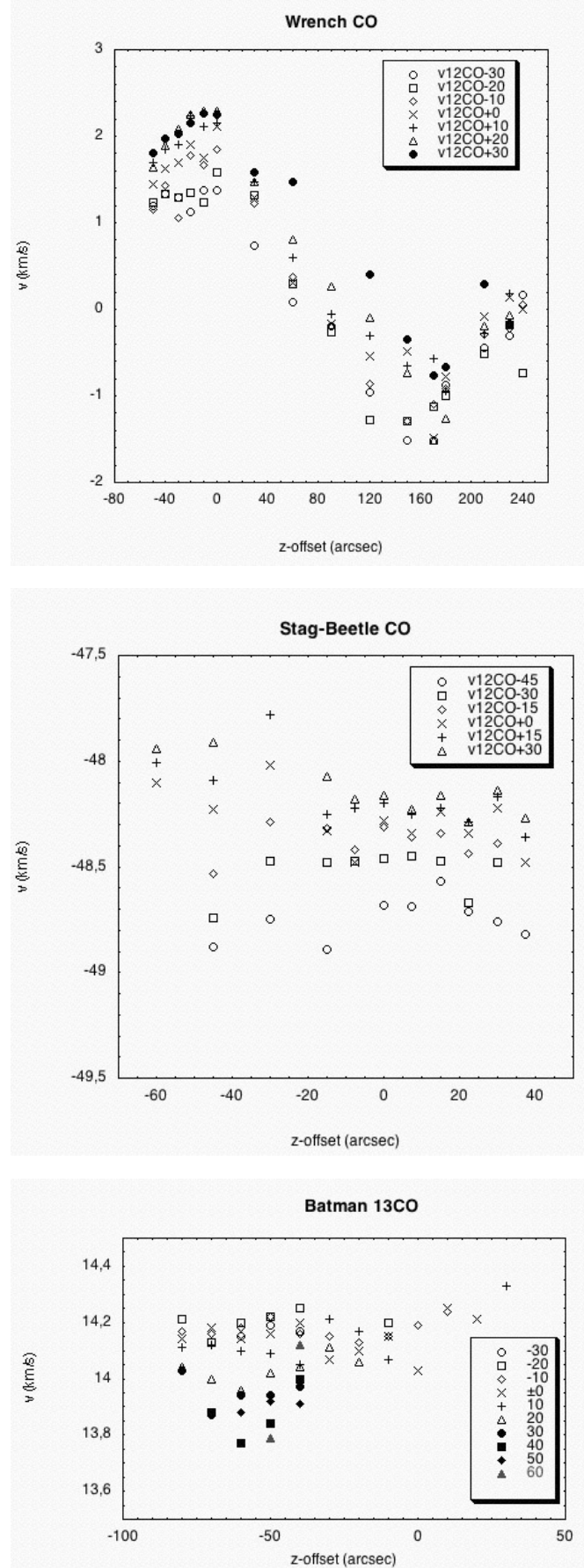

Fig. 11. Top: ${ }^{12} \mathrm{CO}$-velocities over the $W$ Trunk from offsets $-30^{\prime \prime}$ to $+30^{\prime \prime}$ in $z$ (format as in Fig. 10). The jaw is to the left, while the outer filamentary "shell" is seen at the right end at velocities of around $+0.2 \mathrm{~km} \mathrm{~s}^{-1}$. Middle: ${ }^{12} \mathrm{CO}$-velocities over the $S B$ Trunk from offsets $-60^{\prime \prime}$ to $+30^{\prime \prime}$ in $z$, and for integrated line intensities $T_{\mathrm{A}}^{*} \geq 0.5 \mathrm{~K}$. The jaw is at the right end. Bottom: ${ }^{13} \mathrm{CO}$-velocities over the $B$ Trunk from offsets $-30^{\prime \prime}$ to $+80^{\prime \prime}$ in $z$. The jaw is at the left end.

of ${ }^{12} \mathrm{CO}$. The $B$ Trunk is best covered in ${ }^{13} \mathrm{CO}$, and these data enter Fig. 11. Also these trunks show velocity gradient components perpendicular to the major trunk axis, and the $W$ Trunk shows a velocity gradient component along the major axis. From least-square fitting of the velocity data from ${ }^{12} \mathrm{CO}$ and ${ }^{13} \mathrm{CO}$, we derived average values of the projected velocity gradient components perpendicular and parallel to the trunk axis, $v_{x}^{\prime}$ and $v_{z}^{\prime}$, respectively (expressed in $\mathrm{km} \mathrm{s}^{-1}$ per steps of $10^{\prime \prime}$ ). Special weight 
Table 2. Dimensions, inclinations, velocities, and masses of the trunks.

\begin{tabular}{lcccc}
\hline \hline & NGC 7822 & IC 1805 & Rosette & DWB 44 \\
& $D Q$ & $S B$ & $W$ & $B$ \\
\hline Width of the trunk [pc] & 0.12 & 0.62 & 0.29 & V-form \\
Width of the head [pc] & 0.15 & 0.83 & 0.71 & 0.30 \\
Length of whole trunk [pc] & $\geq 1.9$ & 1.9 & 3.5 & 0.9 \\
Inclination & $\sim 35^{\circ}$ & $\sim 80^{\circ}$ & $\sim 35^{\circ}$ & $\sim 45^{\circ}$ \\
$v_{\text {rad }}\left[1 . \mathrm{s.r.} . \mathrm{km} \mathrm{s}^{-1}\right]$ & -16.4 & -48.3 & +0.3 & +14.2 \\
$v_{x}^{\prime}$ in steps of $10^{\prime \prime}\left[\mathrm{km} \mathrm{s}^{-1}\right]$ & $0.17^{*}$ & 0.10 & 0.16 & -0.05 \\
$v_{z}^{\prime}$ in steps of $10^{\prime \prime}\left[\mathrm{km} \mathrm{s}^{-1}\right]$ & 0.05 & $\leq 0.02$ & 0.17 & $\sim 0$ \\
$v_{\text {rot }}^{\prime}\left[\mathrm{km} \mathrm{s}^{-1}\right.$ per $\left.0.1 \mathrm{pc}\right]$ & 0.7 & 0.09 & 0.4 & -0.2 \\
Period [yrs $\left./ 10^{6}\right]$ & 0.8 & 6.9 & 1.5 & 3.1 \\
$v_{\text {axis }}^{\prime}\left[\mathrm{km} \mathrm{s}^{-1} \mathrm{pc}^{-1}\right]$ & 0.85 & $\leq 0.8$ & 1.7 & $\sim 0$ \\
$v_{\text {turb }}\left[\mathrm{km} \mathrm{s}{ }^{-1}\right]$ & $\leq 0.4$ & $\leq 0.5$ & $\leq 0.4$ & $\leq 0.2$ \\
$M_{\mathrm{CO}}\left(\right.$ head/jaw) $\left[M_{\odot}\right]$ & 5.7 & 28.6 & 10.5 & 1.9 \\
$M_{\mathrm{CO}}\left(\right.$ body) $\left[M_{\odot}\right]$ & 3.5 & $9.6^{* *}$ & $6.5^{* *}$ & 0.5 \\
$M_{\mathrm{CO}}$ (total) $\left[M_{\odot}\right]$ & 9.2 & 38.2 & 17.0 & 2.4 \\
\hline$*$ Corrected for beam-filling effects; ${ }^{* *}$ derived from ${ }^{12} \mathrm{CO}$. &
\end{tabular}

was given to regions inside the visible borders of the trunk, and positions with only weak signals were avoided. Linear leastsquare fits to sets of points parallel to the trunk axis at a given offset from the axis show that to a first approximation the velocity across a trunk changes linearly with offset, and approximately with the same amount at different positions along the main trunk axis. This is true in particular for positions within the visible trunk boundary. Such a velocity pattern is consistent with the trunks rotating as rigid bodies.

The $W$ Trunk shows remarkably similar $v_{x}^{\prime}$ :s along the trunk in spite of the fact that the gradient component along the major axis is broken both at the beginning of the jaw and at the connections to the outer shell structure. For the $S B$ Trunk, the $v_{x}^{\prime}$ :s are lowest on the eastern side. Part of this may be due to beamfilling effects at the eastern edge of the trunk, but it appears as if the western extension of the trunk moves at a higher speed than the eastern part. The projected velocities derived for the "trunk neck" ( $z$-offsets $\leq-30$ ) show a larger scatter, but here the signals are weak. The $B$ Trunk is connected to the exterior shell by two diffuse but thin threads, which have about equal radial velocity, but here the signals are weak. Figure 12 shows radial velocity at different offsets from the trunk axis over the jaw region. This velocity gradient is smooth and linear and similar for ${ }^{12} \mathrm{CO}$ and ${ }^{13} \mathrm{CO}$. The values of $v_{x}^{\prime}$ and $v_{z}^{\prime}$ enter Table 2. Negative $v_{x}^{\prime}$ is assigned for anti-clock wise rotation when the trunk is viewed from the connecting shell.

The broadest line profiles are found in the IC 1805 and Rosette regions with line widths (FWHM) ranging between 1.9 to $2.7 \mathrm{~km} \mathrm{~s}^{-1}$ for ${ }^{12} \mathrm{CO}$, and 1.2 to $2.0 \mathrm{~km} \mathrm{~s}^{-1}$ for ${ }^{13} \mathrm{CO}$. The DWB 44 region has the narrowest lines with line widths $\leq 1.1 \mathrm{~km} \mathrm{~s}^{-1}$ for both CO transitions. The line profiles are broadened by thermal motions and velocity shifts due to velocity gradient components across the trunks, projected in the line-ofsight. Turbulent motions may also play a role; and to set limits on the average turbulent velocity, $v_{\text {turb}}$, we first consider the narrowest line profiles, usually from positions just inside the visible trunk borders. The thermal broadening is negligible, and we found that the influence of parallel and perpendicular velocity gradient components on the line broadening is small. Therefore, the FWHM of the narrowest lines, $\langle\Delta v\rangle$, were used to determine the maximum possible values of $v_{\text {turb }}=\langle\Delta v\rangle /(8 \ln 2)^{1 / 2}$, which enter Table 2.

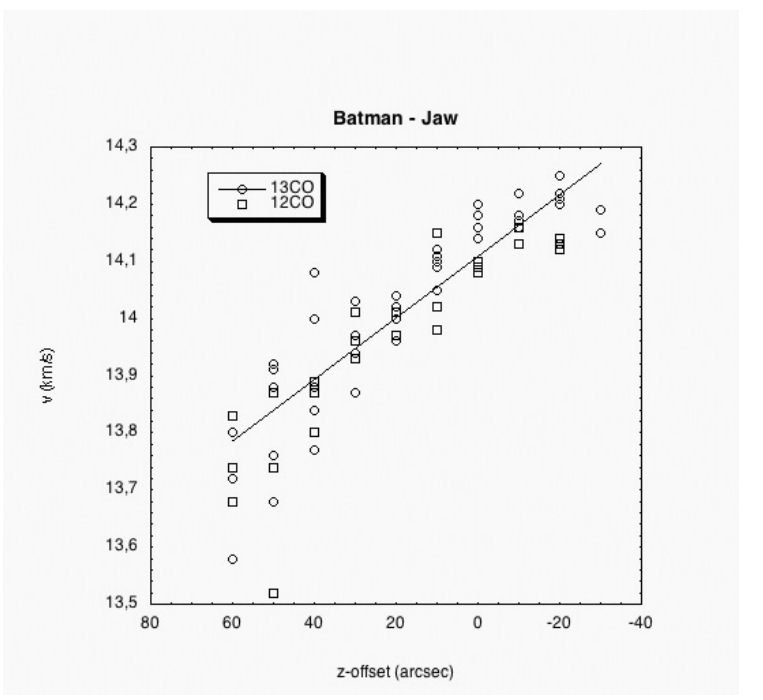

Fig. 12. Velocity versus offset in $x$ over the jaw region of the $B$ Trunk in DWB 44. To a first approximation the velocity gradient components perpendicular to the trunk axis are linear and similar for all offsets in $z$, both for ${ }^{12} \mathrm{CO}$ and ${ }^{13} \mathrm{CO}$.

We assign average radial velocities, $v_{\text {rad }}$, of the trunks in Table 2 in local standard-of-rest velocities. When compared to the central velocities of the regions as derived by others (see Table 1, not measured for DWB 44), one finds that the trunks are blue-shifted in all cases. We infer that the trunks are moving away from the centres of the $\mathrm{H}$ II regions, also consistent with the conclusion above that all trunks are located on the near side of the bubbles.

\subsection{Inclinations}

To derive the true rotational velocity and the significance of a velocity gradient component along a given trunk, we need to correct for the inclination of the trunk, and this angle we define as the angle between the trunk axis and the line-of-sight. The inclination cannot be measured very precisely, but we have made estimates based on the location of the roots (where the trunks are connected to their shells) relative to the centre of the bubble and its periphery. For the inclination angle $i$ given in Table 2, 
we assume that the trunk is pointing radially to the centre of a spherical bubble. The thus derived values of $i$ are uncertain, and it is difficult to assign errors or ranges. It is also difficult to define exactly how a given trunk is connected to the outer shell on the surface of these more or less spherical bubbles. The Rosette Nebula and NGC 7822 are rather regular, and for the former our value of $i$ does not differ much from the $45^{\circ}$ assumed by Schneps et al. (1980).

The $S B$ Trunk has its roots in a massive concentration that intrudes into the bubble from its periphery. Since the radio emission also follows the borders of this dust intrusion, it appears that the trunk and its massive root/shell have hindered the expansion of the ionized gas in this direction. In this case we see the trunk on the plane of the sky, and one should expect $i \approx 90^{\circ}$. However, as noted above, the trunk appears to be blue-shifted relative to the central velocity of the bubble, indicating that the trunk is located on the near side, which is also supported by the extinction measures (see section above). We have assumed an $i=80^{\circ}$ in Table 2. The situation is even less clear for DWB 44, but we have tried to define a morphology of the $\mathrm{H}$ II region from existing radio maps and optical images. The value entering Table 2 is therefore very uncertain.

We then correct for aspect and compute the rotational velocity $v_{\text {rot }}^{\prime}=v_{x}^{\prime} / \sin i$ expressed in $\mathrm{km} \mathrm{s}^{-1}$ per steps of $0.1 \mathrm{pc}$ and the velocity gradient component along the major trunk axis $v_{\text {axis }}^{\prime}=v_{z}^{\prime} \operatorname{tg} i$ expressed in $\mathrm{km} \mathrm{s}^{-1} \mathrm{pc}^{-1}$. For the $S B$ Trunk we can only assign an upper limit to $v_{z}^{\prime}$.

All these data are given in in Table 2 together with the dimensions of the trunks and their heads, where the lengths are corrected for inclination. Also included are the derived periods of rotation. Since all trunks are inclined and the trunks are connected to the outer shells, we exclude the possibility that any velocity gradient component $v_{\text {axis }}^{\prime}$ is related to rotation of the trunks along the $z$-axis. The positive values of $v_{z}$ indicate that the trunks in question are stretching along the major axes.

We conclude that the observed velocity patterns indicate that all our trunks are rotating as rigid (or almost rigid) bodies, and in addition at least two trunks are stretching. The rotational periods of our elephant trunks are comparable to the ages of their central clusters.

\subsection{Masses}

Column densities and masses of the trunks can be estimated from both our $\mathrm{CO}$ data and from the optical extinction derived from our $\mathrm{H} \alpha$ images.

To estimate masses we use formulas that apply to the ${ }^{13} \mathrm{CO}(1-0)$ transition. The corresponding column density, $N_{13}$, is derived from the LTE-formula (see, e.g., Nikolić et al. 2001):

$N_{13}\left[\mathrm{~cm}^{-2}\right]=4.72 \times 10^{13} F\left(T_{\mathrm{ex}}\right) f(\tau) I_{13}$,

where $F\left(T_{\text {ex }}\right)$ is the excitation temperature dependence, $f(\tau)$ the optical depth correction defined as $\left\langle\tau_{13}\right\rangle /\left(1-\mathrm{e}^{-\left\langle\tau_{13}\right\rangle}\right)$, and $I_{13}$ the integrated main-beam brightness temperature $\left[\mathrm{K} \mathrm{km} \mathrm{s}^{-1}\right]$. Assuming a ${ }^{12} \mathrm{CO} /{ }^{13} \mathrm{CO}$ ratio of 75 , i.e. equal to the ${ }^{12} \mathrm{C} /{ }^{13} \mathrm{C}$ isotopic ratio according to Wilson \& Rood (1994), and an intensity ratio of the ${ }^{12} \mathrm{CO}$ and ${ }^{13} \mathrm{CO}$ emission, $I_{12} / I_{13}$, in the range $2.5-10$, the optical depth correction is $f\left(\tau_{13}\right)=1.15 \pm 0.1$. This range of the intensity ratios covers our data, we fix $f\left(\tau_{13}\right)$ to 1.15 . In the interval $10 \mathrm{~K}<T_{\mathrm{ex}}<30 \mathrm{~K}, F\left(T_{\mathrm{ex}}\right)$ is between 20 and 40. Applying a column density ratio between $\mathrm{H}_{2}$ and ${ }^{13} \mathrm{CO}$ of $4.8 \times 10^{5}$ (Dickman \& Clemens 1983) and adding $35 \%$ for $\mathrm{He}$, the total mass of a cloud can be expressed as

$M\left[M_{\odot}\right]=1.35 \times 10^{-11} F\left(T_{\mathrm{ex}}\right) D^{2} A\left\langle I_{13}\right\rangle$,

where $D[\mathrm{pc}]$ is the distance, $A\left[\operatorname{arcsec}^{2}\right]$ is the cloud area, and $\left\langle I_{13}\right\rangle$ is the average integrated ${ }^{13} \mathrm{CO}$ intensity of the area. In cases where only ${ }^{12} \mathrm{CO}$ data are available, we used a ${ }^{12} \mathrm{CO} /{ }^{13} \mathrm{CO}$ intensity ratio, defined in nearby positions, to convert to ${ }^{13} \mathrm{CO}$ intensities. This applies to the bodies of the trunks in IC 1805 and the Rosette Nebula, for which we used $I_{12} / I_{13}$ ratios of 7 and 12 , respectively. The thus derived masses expressed in $M_{\odot}$, of the radio-covered parts of each trunk enter Table 2.

On the NOT images the residual intensity for each pixel within a trunk relative to the interpolated bright background relates to the extinction due to dust in the trunk at $\lambda 6563 \AA$. However, we do not know how much each of the residual intensities is due to foreground $\mathrm{H} \alpha$ emission from the $\mathrm{H}$ II regions engulfing the trunk. Two extreme cases considered: practically all of the residual intensities in a trunk are caused by foreground emission, or there is no foreground emission at all. This sets a range of possible extinctions of the trunks. Using standard procedures we derive the total mass over the radio-covered areas for the two extreme cases.

We find that the masses derived from $\mathrm{CO}$ emission are larger by factors around 2 than the average values derived from extinction. Part of the difference is related to the fact that the radio maps cover thin extended gas located outside the optical trunk boundaries. However, as suggested by Harjunpää \& Mattila (1996), the mass ratio of gas to dust can be higher in regions of active star formation, which could explain part of the discrepancy found.

We note that the Schneps et al. (1980) estimate of the total mass of the southern part of the $W$ Trunk agrees with our estimate well. The subsequent analysis relies on the CO masses. Assuming the trunks to also be symmetric in shape in the 3rd dimension, for instance by assuming cylindric geometries for the main bodies of the trunks, one can derive the average number densities in the trunks. These densities are of the order of $10^{4} \mathrm{~cm}^{-3}$, except for the $S B$ Trunk and the body of the $W$ Trunk with $10^{3} \mathrm{~cm}^{-3}$, which also explains why their ${ }^{13} \mathrm{CO}$ lines are weak. However, the trunks are composed of dense sub-filaments embedded in thinner gas. The $W$ Trunk shows a remarkably complex network of such sub-filaments, and we estimate their densities from extinction measurements across the main trunk to $\sim 8$ times that of the surrounding medium. The pronounced filaments have a volume filling factor of typically 0.04 , which implies that they carry about $1 / 4$ of the total trunk-body mass. The $D Q$ Trunk, on the other hand, appears to be less filamentary.

\section{Discussion}

From our observations we have deduced that these elephant trunks have been accelerated outwards from the centres of the $\mathrm{H}$ II regions in which they reside. An important result is that all four trunks rotate about their axes. The period of rotation is of the same order as the age of the adherent star clusters. In addition, at least two trunks are stretching along the major axis. We now discuss how these results can be interpreted in terms of physical and evolutionary causes.

Table 3 provides data on the properties of the warm plasma surrounding the trunks and total luminosities of the central clusters, $L_{\mathrm{cl}}$. Some references quoted in the table provide information on the spatial variation in electron temperature, $T_{\mathrm{e}}$, electron 
Table 3. Physical quantities of the environments.

\begin{tabular}{lccccl}
\hline \hline & NGC 7822 & IC 1805 & Rosette & DWB 44 & Ref. \\
& $D Q$ & $S B$ & $W$ & $B$ & \\
\hline$T_{\mathrm{e}}[\mathrm{K}]$ & 6400 & $6000^{*}$ & 5800 & $6000^{*}$ & $1-4$ \\
$n_{\mathrm{e}}\left[\mathrm{cm}^{-3}\right]$ & 30 & $10^{*}$ & 10 & $10^{*}$ & $1,3-7$ \\
$v_{\exp }\left[\mathrm{km} \mathrm{s}^{-1}\right]$ & $\leq 7$ & 13.6 & 20 & - & $1,2,8-12$ \\
$\Delta v\left[\mathrm{~km} \mathrm{~s}^{-1}\right]$ & - & $\sim-0.5$ & -1.5 & - & 1 \\
$L_{\mathrm{cl}}\left[10^{5} L_{\odot}\right]$ & 8.5 & 43 & 43 & - & $1,13-15$ \\
\hline
\end{tabular}

* Assumed value.

References: 1. This work; 2. Pedlar (1980); 3. Tereby et al. (2003); 4. Celnik (1985); 5. Harten et al. (1981); 6. Krymkin (1978); 7. Menon (1962); 8. Georgelin \& Georgelin (1970); 9. Dickel et al. (1980); 10. Smith (1973); 11. Fountain et al. (1979); 12. Schneps et al. (1980); 13. Yang \& Fukui (1992); 14. Massey et al. (1995); 15. Cox et al. (1990).

density, $n_{\mathrm{e}}$, and expansion velocity, $v_{\text {exp }}$, of the $\mathrm{H}$ II region, from which we infer the state in the vicinity of the trunks. Also given is the difference in velocity, $\Delta v$, (corrected for aspect), between the centre of the head/top and the surrounding, expanding gas. In the case of the $S B$ Trunk in IC 1805 with an inclination close to $90^{\circ}$, the value of $\Delta v$ is uncertain. We adopted a central velocity of $-46 \mathrm{~km} \mathrm{~s}^{-1}$, which is the mean velocity of $\mathrm{H}$ gas in the bubble (Georgelin \& Georgelin 1970). However, the cluster velocity is considerably different from this, as measured by Dambis et al. (2001) and Hagen (1970). Relative to the cluster, the $S B$ Trunk moves at very high speed if inclined by $80^{\circ}$. However, the motion of the molecular complex, and the H II region may have detached from the cluster motion (see Sect. 2).

Total number densities were derived by assuming a cosmic chemical abundance and a fractional ionization of He I of 0.1 in all regions, as determined for certain locations in NGC 7822 by Pedlar (1980) and in the Rosette Nebula by Celnik (1985). Turbulent velocities were determined for the Rosette Nebula by Viner et al. (1979), who found velocities of up to $37 \mathrm{~km} \mathrm{~s}^{-1}$. However, for the northwestern region, where the $W$ Trunk is located, they obtained $\leq 7 \mathrm{~km} \mathrm{~s}^{-1}$.

From the compiled parameters, we can estimate the external pressures on three of the trunks, and compare them to the internal pressures derived from the trunk parameters listed in Table 2. We find that the external gas pressures amount to a few times $10^{-11}$ dyn $\mathrm{cm}^{-2}$, which is larger than or of the same order as the internal gas pressures.

The area at the head of a trunk facing the centre of the bubble is also subject to radiation pressure generated mainly by the most luminous stars in the central clusters. In the case of the Rosette Nebula, half of the cluster luminosity comes from two bright $\mathrm{O}$ stars, which are offset from the centre in the opposite direction from the $W$ Trunk. When also considering such geometries, we conclude that the external radiation pressures are a few times $10^{-11} \mathrm{dyn} \mathrm{cm}^{-2}$ for the three trunks in question, i.e. comparable to the external gas pressure. This radiation pressure is by itself sufficient to accelerate our trunk heads over the observed distance over the time since the stars were formed.

Our trunks have already gained high velocities in the expanding plasma, and the ram pressures can be estimated to be low $\left(\leq 10^{-12} \mathrm{dyn} \mathrm{cm}^{-2}\right)$ and therefore negligible. However, closer to the stellar cluster the expanding plasma could once have dominated the shaping and acceleration of the clumpy shell from which the trunks developed. The interaction between stellar winds and the surrounding plasma is expected to produce shocked inner zones of high temperature. Judging from the X-ray images of the Rosette Nebula discussed by Townsley et al. (2003) the system of trunks is, presently, outside the central $10 \mathrm{MK}$ gas surrounding the centre.

\subsection{Rotation}

The rotational velocities derived for the trunks indicate that the angular velocity along each of the trunks is fairly constant (within the errors of the measurements). This means that the trunks rotate more or less as solid bodies. If, for the sake of simplicity, the trunks are considered as homogeneous, solid cylinders spinning about their axes, one finds that their angular momenta (from now on SI units are used) are in the range $I \sim 3 \times 10^{48}-2 \times 10^{50} \mathrm{~kg} \mathrm{~m}^{2} \mathrm{~s}^{-1}$, where the upper value refers to the $W$ and $S B$ Trunks. This may be compared with the angular momentum $\sim 10^{45} \mathrm{~kg} \mathrm{~m}^{2} \mathrm{~s}^{-1}$ of a $10 M_{\odot}$ main sequence star spinning at typically $200 \mathrm{~km} \mathrm{~s}^{-1}$. The rotation of e.g. the W Trunk, which has approximately the same mass as this star, thus represents an angular momentum that is at least $10^{5}$ times larger than that of the star.

It is also possible to estimate the kinetic energy connected with the rotation of the trunks by means of the same simple cylindrical model. This energy is found to be in the interval $W_{r}=8 \times 10^{34}-1 \times 10^{37} \mathrm{~J}$ for the four trunks considered. Again, the $W$ Trunk accounts for the largest value. The rotational energies are then of about the same order as the total thermal energies $\left(\sim 10^{36} \mathrm{~J}\right)$.

\subsection{Magnetic fields}

As concluded by CGK02 and CGK03, the magnetic field is likely to play an important role in structuring and confining the filamentary trunks. Little is known about the magnetic fields in trunks from an observational point of view, and we must rely on other methods when estimating typical field strengths. Since the magnetic filaments in a trunk are expected to be formed in the molecular cloud from where the $\mathrm{H}$ II region was formed, it is natural to start from what is known about the magnetic field in such clouds. Measurements and magnetic and virial equilibrium considerations show that the strength of the total magnetic field can be expressed as

$B_{0}=k_{1} n^{1 / 2}$,

where $n$ is the gas number density and $k_{1}=1.6 \times 10^{-13}$ in units of $\left[\mathrm{T} \mathrm{m}^{3 / 2}\right]$ (see e.g. Myers \& Goodman 1988). When a magnetic filament is formed from compression of elongated cloudlets, the longitudinal component of the field must increase to the same degree as the matter density, since the magnetic field is expected to be well frozen-in to the cloud matter. This means that the magnetic field will be larger than $B_{0}$ as given above. If, for instance, the density in the filament is increased by a factor of ten, the filamentary magnetic field will be larger than $B_{0}$ by a factor of about three. Another complication is that matter may be displaced along the magnetic field lines so that the density varies 
along the flux tube without a corresponding variation in the field. This could be the case in the elephant trunks where matter near the head can slide along the field lines towards the head as the H II region expands. Then $B_{0}$ in Eq. (3) should be thought of as a lower limit of the mean magnetic field in the trunk or filament.

It is interesting to compare the rotational energy and the magnetic energy of the trunks. The magnetic energy of a trunk may be found by integrating the magnetic energy density over the trunk volume

$W_{m}=\iiint \frac{B^{2}}{2 \mu_{0}} \mathrm{~d} V$

from which we derive a lower limit of the magnetic energy

$W_{m 0}=\frac{k_{1}^{2} M_{t}}{2 \mu_{0} \bar{m}}$,

where

$M_{\mathrm{t}}=\iiint n \bar{m} \mathrm{~d} V$

is the trunk mass. With a mean mass of the particles of $\bar{m}=$ $4 \times 10^{-27} \mathrm{~kg}$, Eq. (5) yields

$W_{m 0}[\mathrm{~J}]=5.1 \times 10^{36} M_{t}\left[M_{\odot}\right]$.

Applying our trunk masses, we find the lower value of the magnetic energies of the trunks to be in the range $W_{m 0}=1 \times 10^{37}-2 \times$ $10^{38} \mathrm{~J}$. In reality the magnetic energy may be larger than $W_{m 0}$ by perhaps an order of magnitude. Hence, we can conclude that the magnetic energy of the trunks is likely to be at least an order of magnitude larger than both the rotational and thermal energies.

In this connection it may also be useful to consider the Alfvén speed $v_{\mathrm{A}}=B /\left(\mu_{0} \rho\right)^{1 / 2}$ in the trunks. Adopting the magnetic field $B_{0}$ again, as given by Eq. (3), and applying the mass density $\rho=n \bar{m}$, one finds a lower limit of the Alfvén speed

$v_{\mathrm{A} 0}=\frac{B_{0}}{\left(\mu_{0} \rho\right)^{1 / 2}}=\frac{k_{1}}{\left(\mu_{0} \bar{m}\right)^{1 / 2}}$

yielding $v_{\mathrm{A} 0}=2.3 \mathrm{~km} \mathrm{~s}^{-1}$. The value of the real Alfvén speed could very well exceed that of $v_{\mathrm{A} 0}$ by a factor of three or more. It is to be noticed that the maximum rotational speeds in the outer parts of the trunks are all lower than the Alfvén speed, as expected.

Finally we note that the filaments inside the trunks may have rather complicated magnetic morphologies, but the magnetic pressure from more ordered magnetic fields of a few tens of $\mu \mathrm{G}$ in the thinner gas surrounding these filaments will not disrupt the trunks. The centrifugal forces at the trunk surfaces are comparatively small, and it is possible that the trunks can be confined by the external pressure forces alone.

\subsection{Evolving trunks}

Many trunks in our NOT material are $V$ - or $Y$-shaped with a mass condensation at the apex pointing at the central stars. We want to emphasize that the $V$-shaped legs are generally composed of two filaments, and not just the effect of projection from different sight-lines through conical shells. Many filaments appear to be wrapped around each other closer to the head. The big trunks contain networks of thin, and very often twisted, subfilaments connecting the head with the outer expanding shell, often through $V$-shaped structures. The filaments are confined over very large distances. The $D Q, W$, and $B$ Trunks all have $V$-shaped legs connecting the trunk to the shell. Along the main body of the $W$ Trunk and possibly closer to the head in the $D Q$ Trunk, these legs are wound up, reminiscent of the $Y$-shape discussed above. The $S B$ Trunk has no distinct $V$-shaped connection to the shell. This big trunk contains some sub-filaments crossing the trunk axis diagonally, and which may represent connections between the base of the trunk and the head. As shown by CGK03, this trunk has many $V$-shaped sub-filaments sticking out like outgrows from the trunk surface.

That many elephant trunks show sub-filaments wound along the major axis is a natural consequence of rotation. Once the massive head rotates, the connecting filaments will wind up, if they are rooted like magnetic rubber bands in the expanding, but not rotating, shell. With the rotation periods as inferred, these trunks have had time to complete nearly one turn. However, the question is when and how the large angular momentum inferred was transferred to the evolving trunks.

In the theory (see CGK02, CGK03), filaments are assumed to be formed in the molecular clouds surrounding young $\mathrm{H}$ II regions with central, hot $\mathrm{OB}$ stars. As the $\mathrm{H}$ II region expands due to the activity of the hot stars, it sweeps up the surrounding molecular gas in a shell. The magnetized filaments, which can be compared to magnetic flux ropes, will also be swept up by the shell, and incorporated into it, if they are not too massive. If, however, there is a mass condensation in a filament, it will lag behind the rest of the filament due to its larger inertia. Since the magnetic field lines must be considered frozen-in to the matter (magnetic Reynolds number $R_{m} \gg 1$ ), the filament has to form a coherent structure. In case the magnetic field lines inside the filament are not too twisted, part of the filament will attain the shape of a $V$ with its apex pointing towards the centre of the $\mathrm{H}$ II region. The condensation is situated at the apex while the upper parts of the $V$ connect to the shell. In the case the magnetic field lines in the filament are twisted beyond a certain critical limit, a completely new situation will arise. The two filamentary branches next to the apex are then twisted into a double helix so that the filamentary structure instead resembles a $Y$. The elephant trunk is identified with the double helix. The mechanism for forming a double helix out of a magnetic rope was verified both from an analogy model (see Fig. 3 in CGK02) and by a numerical model (CGK03).

The discovery that the trunks are rotating, sheds new light on how many structures present in elephant trunks can arise. The trunks are continuously stretching and changing shape thereby implying that electric currents are also generated, which together with the magnetic field play an important role in sculpturing the trunks. We have demonstrated that the total magnetic energy in a trunk is a dominant energy source and that most likely magnetic forces control the shaping and confinement of sub-filaments. Along this line, we now presume that the origin of rotation can be related to the formation of compressed magnetic filaments that were present in the parent molecular core around the young, compact $\mathrm{H}$ II region.

The theory of magnetically twisted trunks, discussed above, so far only accounts for the stationary state. In order to understand the time development of the trunks, a dynamic model is needed. Under certain conditions, when the time development of the trunks is sufficiently slow, one may consider the evolution of the trunks as a series of stationary states. The condition for this is that the relative change in the trunks during the propagation time of an Alfvén wave through the system should be small. Fortunately, this condition seems to be reasonably well fulfilled in the trunks considered. Hence, we may use the 
double helix model for obtaining a fairly good picture of how the trunks became twisted. From the double helix model we know that if a double helix has already been formed through the twisting of a filament, a further twist of the filament, of say one turn, will result in a twist of half a turn of the double helix. One may consider this a result of the constancy of the helicity of the magnetic field $H=\langle\boldsymbol{A} \cdot \boldsymbol{B}\rangle$ where $\boldsymbol{A}$ is the vector potential of the magnetic field $\boldsymbol{B}$ and $\langle\cdot\rangle$ denotes the volume average over the filament (CGK03 and references therein). The new twisting of the double helix will take place at the position where the double helix is split up into two filamentary legs. This means that the double helix will rotate more or less as a solid body with the same angular speed along the major axis, which is consistent with our observations.

Elephant trunks are photo-evaporating, as manifested in the bright rims. The trunks also pinch off globule-like clumps such as seen surrounding the $W$ Trunk. Moreover, many trunks have jaws connected to the heads (see Figs. 2-4). The jaws are in all cases extremely filamentary and composed of very thin, twisted, and often distinctly helical strings. The ends of the jaws in our material rotate at high speed about the trunk axis in accordance with the solid body rotation. In the case of the $B$ Trunk, it appears as if the jaw is shaped from a slab of gas that is now swinging out as a wing under the disruptive forces of winds and radiation in the thin but warm environment. This whole slab rotates as a rigid body. We have numerous examples in our NOT material of trunks where blobs or filaments have detached from the trunks. Figure 1 shows how a big filamentary block has detached from the head of the $D Q$ Trunk. It is wrapped and rotated around the trunk. Another big trunk is found to the right of this image, where the head is breaking up in a similar way. Hence, the trunks are eroding with time, and were more massive at earlier phases.

We have found that the double helix mechanism could explain how angular momentum is deposited into filaments in the parent clouds and how elephant trunks develop twisted $V$ - and $Y$-shaped forms. However, at present it is difficult to speculate on the details of this initial angular momentum transfer; and even though the trunks are still twisted, it is not clear that they are all double helices at these late phases when substantial erosion has occurred. The jaws may be a natural result of eroded, closed loops at the apex of the trunks.

Another pillar, namely the Horseshead Nebula, was recognised by Pound et al. (2003) to possess smooth gradient components in radial velocity. The velocity pattern shown in their Fig. 7 is very reminiscent of what we have found for our rotating trunks, and recently Hily-Blant et al. (2005) have concluded that one subfilament, called the Neck, is rotating. They also find that the angular velocity varies with position in the trunk probably due to the presence of a rather massive clump located in the middle of the filament.

Our resulting picture is that rotation is a common property of elephant trunks and that magnetic forces play a leading role in the evolution of these beautiful but extremely complex systems. Our results also demonstrate how angular momentum can be removed from regions of star formation.

\section{Conclusions}

Four cold, dark, and filamentary pillars were selected from our collection of $\mathrm{H} \alpha$ images of $\mathrm{H}$ II regions and were mapped mainly in the emission lines of ${ }^{12} \mathrm{CO}(1-0)$ and ${ }^{13} \mathrm{CO}(1-0)$. These socalled elephant trunks are connected with $V$-shaped filaments to outer, expanding molecular shells surrounding the expanding $\mathrm{H}$ II regions. All trunks show signs of being twisted, and they contain complicated networks of filamentary substructures, which in turn are twisted and also can have helical shapes. From our optical and radio observations of these trunks, we derived masses and average densities of the trunks in question, and we made a detailed analysis of the smooth gradient components in radial velocity discovered both along and across the major axes of the trunks.

The trunks have masses of $2-38 M_{\odot}$, and average densities of the order of $10^{3}-10^{4} \mathrm{~cm}^{-3}$. Most of the mass is concentrated in the sub-filaments and particularly in the rather massive heads that face the centres of the H II regions. We infer that all the trunks we observed are located on the near side of the more or less spherical $\mathrm{HII}$ bubbles and that they are moving out from the centres. The gradient component in radial velocity along the major axes indicate that the trunks are stretching in the sense that the massive head is lagging behind in the general expansion. This implies that the trunks once formed closer to the centres of the parent molecular clouds contain filamentary blocks of gas with mass condensation. They were subsequently accelerated outwards and sculptured through the influence of expanding warm plasma, radiation pressure from the central young cluster, and electromagnetic forces. The trunks are eroding and losing mass with time.

An important discovery is that all four trunks turn out to rotate about their major axes and that the trunks, to the first approximation, rotate as solid bodies. With estimates of the inclinations of the trunks to the line-of-sights, we conclude that the orbital periods are 0.8-6.9 million years, similar to the age of the adherent stellar clusters. The corresponding angular momentum is very large, $3 \times 10^{48}-2 \times 10^{50} \mathrm{~kg} \mathrm{~m}^{2} \mathrm{~s}^{-1}$, and the total rotational energies are in the range $8 \times 10^{34}-1 \times 10^{37} \mathrm{~J}$, which is the same order as the total thermal energies.

The magnetic field strengths in the trunks are not known to any precision. We have estimated the total magnetic energies to be in the range $1 \times 10^{37}-2 \times 10^{38} \mathrm{~J}$, which is at least an order of magnitude higher than both the rotational and thermal energies. The magnetic fields are frozen-in to the matter, and the fields are condensed and re-structured when the trunks evolve. One can envision how the structure of the trunks with their twisted subfilaments is dominated and controlled by magnetic forces, and it can also explain how the thin but very extended sub-filaments can be confined. The twisted appearance of individual trunks can be understood as a consequence of rotation, and on the whole they can be confined by the kinetic pressures from the surrounding warm plasma.

The huge reservoirs of magnetic energy can explain how the elephant trunks once gained their large angular momenta, and we outline a mechanism for explaining the formation of rotating trunks that is based on an extension of a theory of elephant trunks developed earlier (e.g. CGK03). Finally, our results point to a new process for removing angular momentum from a parent cloud after the formation of an $\mathrm{H}$ II region.

Acknowledgements. We thank Helmuth Kristen who participated in the preparation of the NOT images, and an anonymous referee for constructive suggestions. This work was supported by the Carl Trygger's Foundation. S. N. acknowledges support of the Chilean Centro de Astrofísica FONDAP No. 15010003 and the Serbian Ministry of Science and Environmental Protection grant No. 136001.

\section{References}

Basu, S., Johnston, D., \& Martin, P. G. 1999, ApJ, 516, 843 Bok, B. J., Olmsted, M., \& Boutelle, B. D. 1949, ApJ, 110, 21 Carlqvist, P., Kristen, H., \& Gahm, G. F. 1998, A\&A, 332, L5 (CKG98) Carlqvist, P., Gahm, G. F., \& Kristen, H. 2002, Ap\&SS, 280, 405 (CGK02) Carlqvist, P., Gahm, G. F., \& Kristen, H. 2003, A\&A, 403, 399 (CGK03) 
Carpenter, J. M., Heyer, M. H., \& Snell, R. L. 2000, ApJS, 130, 381

Celnik, W. E. 1985, A\&A, 144, 171

Cohen, M., \& Kuhi, L. V. 1976, ApJ, 210, 365

Cox, P., Deharveng, L., \& Leene, A. 1990, A\&A, 230, 181

Dambis, A. K., Mel'nik, A. M., \& Rastorguev, A. S. 2001, Astron. Lett., 27, 58

Dickel, H. R., \& Wendker, H. 1977, A\&AS, 29, 209

Dickel, H. R., Wendker, H., \& Bieritz, J. H. 1969, A\&A, 1, 270

Dickel, H. R., Dickel, J. R., Wilson, W. J., \& Werner, M. W. 1980, ApJ, 237, 711

Dickman, R. L., \& Clemens, D. P. 1983, ApJ, 271, 143

Frieman, E. A. 1954, ApJ, 120, 18

Fountain, W. F., Gary, G. A., \& O'Dell, C. R. 1979, ApJ, 229, 971

Gahm, G. F. 2003, in Recent Research Developments in Astrophysics, Research signposts, Kerala, India, 1, 117

Garcia-Segura, G., \& Franco, J. 1996, ApJ, 469, 171

Georgelin, Y. P., \& Georgelin, Y. M. 1970, A\&A, 6, 349

Guiliani, J. L. 1979, ApJ, 233, 280

Graham, D. A., Haslam, C. G., Salter, C. J., \& Wilson, W. E. 1982, A\&A, 109 , 145

Guetter, H. H., \& Vrba, F. J. 1989, AJ, 98, 611

Gyul'budagyan, A. L., \& Akopyan, V. A. 1990, Afz, 33, 395

Gyul'budagyan, A. L., Oganyan, G. B., \& Akhverdyan, L. G. 1994, Afz, 37, 577

Hagen, G. 1970, A\&A, 146, 109

Harjunpää, P., \& Mattila, K. 1996, A\&A, 305, 920

Harten, R. H., Goss, W. M., Matthews, H. E., \& Israel, F. P. 1981, A\&A, 103, 50

Herbig, G. H. 1974, PASP, 86, 604

Hester, J. J., Scowen, P. A., Sankrit, R., et al. 1996, AJ, 111, 2349

Hily-Blant, P., Teyssier, D., Philipp, S., \& Güsten, R. 2005, A\&A, in press

Krymkin, V. V. 1978, Ap\&SS, 54, 187

Lada, C. J., Elmegreen, B. G., Cong, H.-I., \& Thaddeus, P. 1978, ApJ, 226, L39

Leisavitz, D., Bash, F. N., \& Thaddeus, P. 1989, ApJS, 70, 731

Liu, T., Janes, K. A., Bania, T. M., \& Phelps, R. L. 1988, AJ, 95, 1122
MacConnell, D. J. 1968, ApJS, 16, 275

Massey, P., Johnson, K. E., \& DeGioia-Eastwood, K. 1995, ApJ, 454, 151

Menon, T. K. 1962, ApJ, 135, 394

Minkowski, R. 1949, PASP, 61, 151

Mirabel, I. F., Rodrigues, I., \& Liu, Q. Z. 2004, A\&A, 422, L29

Myers, P. C., \& Goodman, A. A. 1988, ApJ, 326, L27

Nikolić, S., Kiss, Cs., Johansson, L. E. B., et al. 2001, A\&A, 367, 694

Normandeau, M., Taylor, A. R., \& Dewdney, P. E. 1997, ApJS, 108, 279

Ogura, K., \& Ishida, K. 1981, PASJ, 33, 149

Ogura, K., \& Sugitani, K. 1998, Pub. Astron. Soc. Australia, 15, 91

Ogura, K., Sugitani, K., \& Pickle, A. 2002, ApJ, 123, 2597

Osterbrock, D. E. 1957, ApJ, 125, 622

Pedlar, A. 1980, PASP, 99, 1050

Pikel'ner, S. B. 1973, Comm. Astrophys., 5, 151

Pikel'ner, S. B., \& Sorochenko, R. L. 1974, SvA, 17, 443

Pottasch, S. 1956, Bull. Astron. Soc. Netherlands, 13, 77

Pound, M. W. 1998, ApJ, 493, L113

Pound, M. W., Reipurth, B., \& Bally, J. 2003, AJ, 125, 2108

Rydbeck, O. E. H., Kollberg, E., Hjalmarsson, A., et al. 1976, ApJS, 31, 333

Schneps, M. H., Ho, P. T. P., \& Barret, A. H. 1980, ApJ, 240, 84

Smith, M. G. 1973, ApJ, 182, 111

Spitzer, L. Jr. 1954, ApJ, 120, 1

Sugitano, K., Fukui, Y., \& Ogura, K. 1991, ApJS, 77, 59

Taylor, A. R., Irwin, J. A., Matthews, H. E., \& Heyer, M. H. 1999, ApJ, 513, 339

Tereby, S., Fich, M., Taylor, R., et al. 2003, ApJ, 590, 906

Townsley, L. K., Feigelson, E. D., Montmerle, T., et al. 2003, ApJ, 593, 874

Viner, M. R., Vallée, J. P., \& Hughes, V. A. 1979, AJ, 84, 1335

Wendker, H. J., Higgs, L. A., \& Landecker, T. L. 1991, A\&A, 241, 551

White, G. J., Nelson, R. P., Holland, W. S., et al. 1999, A\&A, 342, 233

Wilson, T. L., \& Rood, R. 1994, ARA\&A, 32, 191

Yang, J., \& Fukui, Y. 1992, ApJ, 386, 618 